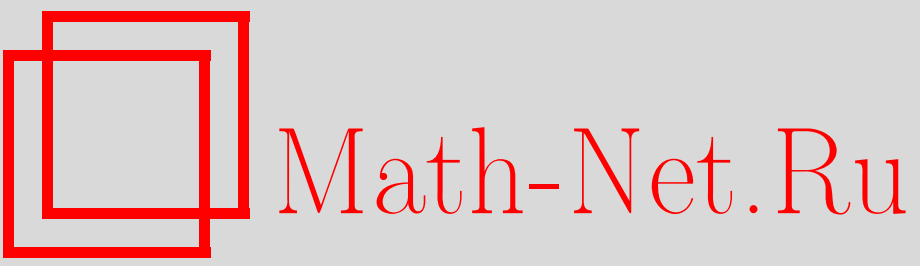

Е. А. Головко, О разрешимости задачи Дирихле для многомерной эллиптической системы в полупространстве, Итоги науки и техн. Сер. Соврем. мат. и ее прил. Темат. обз., 2020, том 183, 52-60

DOI: https://doi.org/10.36535/0233-6723-2020-183-52-60

Использование Общероссийского математического портала Math-Net.Ru подразумевает, что вы прочитали и согласны с пользовательским соглашением

http: //www.mathnet.ru/rus/agreement

Параметры загрузки:

IP: 52.6 .47 .48

26 апреля 2023 г., 18:00:14 


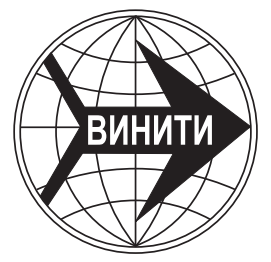

ИТОГИ НАУКИ И ТЕХНИКИ.

Современная математика и ее приложения.

Тематические обзоры.

Том 183 (2020). C. $52-60$

DOI: $10.36535 / 0233-6723-2020-183-52-60$

УДК 517.956 .2

\title{
О РАЗРЕШИМОСТИ ЗАДАЧИ ДИРИХЛЕ \\ ДЛЯ МНОГОМЕРНОЙ ЭЛЛИПТИЧЕСКОЙ СИСТЕМЫ \\ В ПОЛУПРОСТРАНСТВЕ
}

\author{
(c) 2020 г. $\quad$ Е. А. ГОЛОВКО
}

\begin{abstract}
АннотАция. В работе рассмотрена задача Дирихле для многомерной эллиптической системы в полупространстве. С помощью преобразования Фурье вопрос о разрешимости рассматриваемой задачи сведен к исследованию одного уравнения с частными производными второго порядка.
\end{abstract}

Ключевые слова: многомерная эллиптическая система, полупространство, задача Дирихле, преобразование Фурье, функция Бесселя.

\section{ON THE SOLVABILITY OF THE DIRICHLET PROBLEM FOR A MULTIDIMENSIONAL ELLIPTIC SYSTEM IN THE HALF-SPACE}

\author{
(c) 2020 E. A. GOLOVKO
}

\begin{abstract}
The Dirichlet problem for a multidimensional elliptic system in the half-space is considered. With the help of the Fourier transform, the problem of the solvability of the problem is reduced to the study of a second-order partial differential equation.
\end{abstract}

Keywords and phrases: multidimensional elliptic system, half-space, Dirichlet problem, Fourier transform, Bessel function.

AMS Subject Classification: 35J57

Важным разделом теории уравнений с частными производными является теория краевых задач для эллиптических уравнений и систем. Среди таких задач наибольший интерес представляют так называемые нефредгольмовы краевые задачи, исследование которых, как правило, сводится к изучению сингулярных интегральных уравнений, причем для этих задач нарушается альтернатива Фредгольма. Благодаря разработанности теории одномерных сингулярных интегральных уравнений краевые задачи для эллиптических уравнений с двумя независимыми переменными в настоящее время полностью изучены, чего нельзя сказать о краевых задачах для эллиптических уравнений с многими независимыми переменными. Ряд важных вопросов в этой области не решен до сих пор, так как нет достаточно общих методов исследования (см. [5]).

Постановка классических граничных задач для уравнения Лапласа связана с физическими приложениями. Эти задачи корректны для любых эллиптических уравнений с частными производными второго порядка. Задача Дирихле для таких уравнений всегда фредгольмова (см. [3]). Уравнения и системы уравнений с двумя независимыми переменными изучены достаточно полно. Гораздо сложнее обстоит дело с эллиптическими уравнениями и системами с многими независимыми переменными. 
Определение 1. Система уравнений в частных производных называется эллиптической по Петровскому, если определитель ее характеристической формы является положительно определенной либо отрицательно определенной формой.

В 1949 г. А. В. Бицадзе привел пример системы двух уравнений второго порядка (см. [1])

$$
\left\{\begin{array}{l}
-\Delta u+2 \frac{\partial}{\partial x}\left(u_{x}+v_{y}\right)=0 \\
-\Delta v+2 \frac{\partial}{\partial y}\left(u_{x}+v_{y}\right)=0
\end{array}\right.
$$

для которой нарушается не только фредгольмовость, но и нетеровость задачи Дирихле. Нетеровость и фредгольмовость задачи понимаются здесь следующим образом (см. [4]).

Определение 2. Граничная задача называется нетеровой, если для ее разрешимости на данные задачи необходимо наложить конечное число $p$ условий типа условий ортогональности, а соответствующая ей однородная задача имеет конечное число $q$ линейно независимых решений. Если же задача нетерова и $p=q$, то такую задачу называют фредгольмовой.

Однородная задача Дирихле для системы (1) с условиями

$$
\left.u\right|_{\Gamma}=0,\left.\quad v\right|_{\Gamma}=0
$$

в круге $K:\left\{x^{2}+y^{2}<1\right\}$ с границей $\Gamma:\left\{x^{2}+y^{2}=1\right\}$ имеет бесконечное множество линейно независимых решений

$$
u=\left(1-x^{2}-y^{2}\right) \varphi_{x}, \quad v=\left(1-x^{2}-y^{2}\right) \varphi_{y},
$$

где $\varphi$ - произвольная регулярная в круге $K$ и непрерывно дифференцируемая в замкнутом круге гармоническая функция (см. [1]).

Таким образом, характер разрешимости классических граничных задач для эллиптических систем уравнений в частных производных существенно сложнее, чем для одного уравнения.

В 1951 г. М. И. Вишик выделил класс систем, для которых классические граничные задачи нетеровы. Это сильно эллиптические системы (см. [2]).

Определение 3. Эллиптическая по Петровскому система называется сильно эллиптической, если симметрическая составляющая ее характеристической матрицы положительно определена либо отрицательно определена, т.е. все ее собственные числа имеют одинаковые знаки.

Однако среди систем, не удовлетворяющих условию сильной эллиптичности, имеются системы, для которых задача Дирихле нетерова (см. [4]). Поэтому вопрос о влиянии структуры системы на разрешимость граничных задач представляется актуальным. Из класса эллиптических систем, не удовлетворяющих условию сильной эллиптичности, наиболее полно исследованы системы с двумя независимыми переменными (см. [1]). Любую сильно эллиптическую систему двух уравнений с частными производными второго порядка с двумя независимыми переменными, непрерывно меняя коэффициенты, можно продеформировать в систему двух отдельных уравнений Лапласа. Систему же, не удовлетворяющую условию сильной эллиптичности, можно продеформировать в системуЁ(1). Системы с многими независимыми переменными, не удовлетворяющие условию сильной эллиптичности, изучены гораздо слабее. ВЁэтом направлении исследованы лишь системы специального вида.

Рассмотрим задачу Дирихле для многомерной системы

$$
-\Delta u_{k}+\sum_{j=1}^{n} \lambda_{k j} \frac{\partial}{\partial x_{j}} \sum_{i=1}^{n} \frac{\partial u_{i}}{\partial x_{i}}=0, \quad k=1, \ldots, n,
$$

в следующей постановке: найти регулярные в полупространстве $G=\left\{x_{n}>0\right\}$ решения системы (3), удовлетворяющие на границе $\Gamma=\left\{x_{n}=0\right\}$ этого полупространства условиям

$$
\left.u_{k}\right|_{x_{n}=0}=f_{k}, \quad k=1, \ldots, n,
$$

где $f_{k}\left(x_{1}, \ldots, x_{n-1}\right)$ - заданные достаточно гладкие функции. 
Введем обозначение

$$
H=\sum_{i=1}^{n} \frac{\partial u_{i}}{\partial x_{i}}
$$

и перепишем (3) в виде

$$
-\Delta u_{k}+\sum_{j=1}^{n} \lambda_{k j} \frac{\partial H}{\partial x_{j}}=0, \quad k=1, \ldots, n .
$$

Продифференцируем $k$-е уравнение системы (6) по $x_{k}$ и сложим полученные результаты:

$$
-\Delta H+\sum_{k, j=1}^{n} \lambda_{k j} \frac{\partial^{2} H}{\partial x_{k} \partial x_{j}}=0
$$

Применим к последнему уравнению преобразование Фурье по переменным $x_{1}, \ldots, x_{n-1}$ :

$$
\left(\lambda_{n n}-1\right) \frac{\partial^{2} \tilde{H}}{\partial x_{n}^{2}}+\sum_{k=1}^{n-1}\left(\lambda_{k n}+\lambda_{n k}\right)\left(-i \xi_{k}\right) \frac{\partial \tilde{H}}{\partial x_{n}}+\left(\sum_{k=1}^{n-1} \xi_{k}^{2}-\sum_{k, j=1}^{n-1} \lambda_{k j} \xi_{k} \xi_{j}\right) \tilde{H}=0 .
$$

Здесь $\tilde{H}\left(\xi_{1}, \ldots, \xi_{n-1}, x_{n}\right)$ - трансформанта Фурье по переменным $x_{1}, \ldots, x_{n-1}$ функции $H\left(x_{1}, \ldots, x_{n}\right)$.

Введем обозначения

$$
\beta^{2}=\frac{1}{\lambda_{n n}-1}\left(\sum_{k, j=1}^{n-1} \lambda_{k j} \xi_{k} \xi_{j}-\sum_{k=1}^{n-1} \xi_{k}^{2}\right), \quad \rho^{2}=\sum_{k=1}^{n-1} \xi_{k}^{2}, \quad \alpha=\frac{1}{\lambda_{n n}-1} \sum_{k=1}^{n-1}\left(\lambda_{n k}+\lambda_{k n}\right) \xi_{k} .
$$

С учетом введенных обозначений уравнение (7) примет вид

$$
\frac{\partial^{2} \tilde{H}}{\partial x_{n}^{2}}-\alpha i \frac{\partial \tilde{H}}{\partial x_{n}}-\beta^{2} \tilde{H}=0 .
$$

Ограниченные на бесконечности решения последнего уравнения можно записать в виде

$$
\tilde{H}=C\left(\xi_{1}, \ldots, \xi_{n-1}\right) e^{h x_{n}},
$$

где $C\left(\xi_{1}, \ldots, \xi_{n-1}\right)$ - произвольная функция, а $h-$ корень уравнения $h^{2}-i \alpha h-\beta^{2}=0$ :

$$
h=\frac{\alpha i-\sqrt{-\alpha^{2}+4 \beta^{2}}}{2} .
$$

Применяя преобразование Фурье к системе (6) и учитывая (9), имеем

$$
\frac{\partial^{2} \tilde{u}_{k}}{\partial x_{n}^{2}}-\rho^{2} \tilde{u}_{k}=C e^{h x_{n}}\left(\sum_{j=1}^{n-1} \lambda_{k j} \xi_{k} i-\lambda_{k n} h\right), \quad k=1, \ldots, n .
$$

Рассмотрим $k$-е уравнение полученной системы. Общее решение каждого уравнения системы (10) можно представить в виде

$$
\tilde{u}_{k}=v_{k}+w_{k}, \quad k=1, \ldots, n,
$$

где $v_{k}$-общее решение $k$-го однородного уравнения, соответствующего $k$-му уравнению системы (10), а $w_{k}$ - частное решение $k$-го неоднородного уравнения системы $(10), k=1, \ldots, n$. Ограниченные на бесконечности решения $k$-го однородного уравнения имеют вид

$$
v_{k}=D_{k}\left(\xi_{1}, \ldots, \xi_{n-1}\right) e^{-\rho x_{n}}, \quad k=1, \ldots, n .
$$

Если искать частные решения $w_{k}$ в виде

$$
w_{k}=F_{k}\left(\xi_{1}, \ldots, \xi_{n-1}\right)\left(e^{h x_{n}}-e^{-\rho x_{n}}\right), \quad k=1, \ldots, n,
$$


из системы (10) находим

$$
-F_{k}\left(h^{2} e^{h x_{n}}-\rho^{2} e^{-\rho x_{n}}\right)+\rho^{2} F_{k}\left(e^{h x_{n}}-e^{-\rho x_{n}}\right)=C e^{h x_{n}}\left(\sum_{j=1}^{n-1} \lambda_{k j} \xi_{k} i-\lambda_{k n} h\right) .
$$

Отсюда получаем

$$
F_{k}=\frac{C}{h^{2}-\rho^{2}}\left(\lambda_{k n} h-\sum_{j=1}^{n-1} \lambda_{k j} \xi_{j} i\right), \quad k=1, \ldots, n .
$$

Таким образом, ограниченные на бесконечности решения системы (10) можно представить в виде

$$
\tilde{u}_{k}=D_{k} e^{-\rho x_{n}}+\frac{C}{h^{2}-\rho^{2}}\left(\lambda_{k n} h-\sum_{j=1}^{n-1} \lambda_{k j} \xi_{j} i\right)\left(e^{h x_{n}}-e^{-\rho x_{n}}\right), \quad k=1, \ldots, n,
$$

где $D_{k}\left(\xi_{1}, \ldots, \xi_{n-1}\right), k=1, \ldots, n$, и $C\left(\xi_{1}, \ldots, \xi_{n-1}\right)$ - произвольные функции. Определим $D_{k}$, используя граничные условия (4) исходной задачи. В терминах преобразования Фурье условия (4) запишутся следующим образом:

$$
\left.\tilde{u}\right|_{x_{n}=0}=\tilde{f}_{k}, \quad k=1, \ldots, n
$$

Следовательно,

$$
D_{k}\left(\xi_{1}, \ldots, \xi_{n-1}\right)=\tilde{f}_{k}\left(\xi_{1}, \ldots, \xi_{n-1}\right), \quad k=1, \ldots, n .
$$

Определим $C\left(\xi_{1}, \ldots, \xi_{n-1}\right)$ из условия $(5)$, которое в терминах преобразования Фурье запишется в виде

$$
-\sum_{k=1}^{n-1} \tilde{u}_{k} \xi_{k} i+\frac{\partial \tilde{u}_{n}}{\partial x_{n}}=C e^{h x_{n}}
$$

Подставляя в последнее уравнение выражения (11) для $\tilde{u}_{k}$, получим

$$
\begin{aligned}
& -\sum_{k=1}^{n-1} \tilde{f}_{k} \xi_{k} i e^{-\rho x_{n}}-\rho \tilde{f}_{n} e^{-\rho x_{n}}+ \\
& +\frac{C}{h^{2}-\rho^{2}}\left[\left(-\sum_{k=1}^{n-1} \lambda_{k n} \xi_{k} h i-\sum_{k, j=1}^{n-1} \lambda_{k j} \xi_{k} \xi_{j}\right)+\left(\lambda_{n n} h^{2}-\sum_{j=1}^{n-1} \lambda_{n j} \xi_{j} h i\right)\right] e^{h x_{n}}+ \\
& +\frac{C}{h^{2}-\rho^{2}}\left[\left(\lambda_{n n} h \rho-\rho \sum_{j=1}^{n-1} \lambda_{n j} \xi_{j} i\right)+\left(\sum_{k=1}^{n-1} \lambda_{k n} h \xi_{k} i+\sum_{k, j=1}^{n-1} \lambda_{k j} \xi_{k} \xi_{j}\right)\right] e^{-\rho x_{n}}=C e^{h x_{n}} .
\end{aligned}
$$

Покажем, что

$$
T \equiv\left[\left(-h i \sum_{k=1}^{n-1} \lambda_{k n} \xi_{k}-\sum_{k, j=1}^{n-1} \lambda_{k j} \xi_{k} \xi_{j}\right)+\left(\lambda_{n n} h^{2}-\sum_{j=1}^{n-1} \lambda_{n j} \xi_{j} h i\right)\right] \equiv h^{2}-\rho^{2} .
$$

Рассмотрим левую часть тождества (13). Так как $h^{2}-\alpha h i+\beta^{2}=0$, или $h^{2}=\alpha h i-\beta^{2}$, имеем

$$
T \equiv\left(-h i \sum_{k=1}^{n-1}\left(\lambda_{k n}+\lambda_{n k}\right) \xi_{k}\right)+\left(-\sum_{k, j=1}^{n-1} \lambda_{k j} \xi_{k} \xi_{j}+\lambda_{n n}\left(\alpha h i+\beta^{2}\right)\right) .
$$

Учитывая введенные выше обозначения (8), преобразуем последнее выражение: 


$$
\begin{aligned}
T \equiv-h i \sum_{k=1}^{n-1}\left(\lambda_{k n}+\lambda_{n k}\right) \xi_{k}-\sum_{k, j=1}^{n-1} \lambda_{k j} \xi_{k} \xi_{j}+ & \\
+ & \frac{\lambda_{n n} h i}{\lambda_{n n}-1} \sum_{k=1}^{n-1}\left(\lambda_{n k}+\lambda_{k n}\right) \xi_{k}+\frac{1}{\lambda_{n n}-1}\left(\sum_{k, j=1}^{n-1} \lambda_{k j} \xi_{k} \xi_{j} \lambda_{n n}-\sum_{k=1}^{n-1} \lambda_{n n} \xi_{k}^{2}\right)= \\
= & \frac{-\left(\lambda_{n n}-1\right)}{\lambda_{n n}-1} h i \sum_{k=1}^{n-1}\left(\lambda_{k n}+\lambda_{n k}\right) \xi_{k}+\frac{-\left(\lambda_{n n}-1\right)}{\lambda_{n n}-1} \sum_{k, j=1}^{n-1} \lambda_{k j} \xi_{k} \xi_{j}+ \\
& +\frac{\lambda_{n n}}{\lambda_{n n}-1} h i \sum_{k=1}^{n-1}\left(\lambda_{k n}+\lambda_{n k}\right) \xi_{k}+\frac{\lambda_{n n}}{\lambda_{n n}-1} \sum_{k, j=1}^{n-1}\left(\lambda_{k j} \xi_{k} \xi_{j}-\lambda_{n n}\right) \xi_{k}^{2}= \\
= & \frac{h i}{\lambda_{n n}-1} \sum_{k=1}^{n-1}\left(\lambda_{n k}+\lambda_{k n}\right) \xi_{k}+\frac{1}{\lambda_{n n}-1} \sum_{k, j=1}^{n-1} \lambda_{k j} \xi_{k} \xi_{j}-\frac{1}{\lambda_{n n}-1} \sum_{k=1}^{n-1} \xi_{k}^{2}+ \\
& +\frac{1}{\lambda_{n n}-1} \sum_{k=1}^{n-1} \xi_{k}^{2}-\frac{\lambda_{n n}}{\lambda_{n n}-1} \sum_{k=1}^{n-1} \xi_{k}^{2}=h \alpha i+\beta^{2}-\sum_{k=1}^{n-1} \xi_{k}^{2}=h^{2}-\rho^{2}
\end{aligned}
$$

Отсюда следует, что

$$
h i \sum_{k=1}^{n-1} \lambda_{k n} \xi_{k}+\sum_{k, j=1}^{n-1} \lambda_{k j} \xi_{k} \xi_{j}=\frac{1}{h^{2}-\rho^{2}} \lambda_{n n} h^{2}-\sum_{j=1}^{n-1} \lambda_{n j} \xi_{j} h i-\left(h^{2}-\rho^{2}\right) .
$$

Подставляя эти выражения в уравнение (12), получим

$$
\begin{aligned}
& -\sum_{k=1}^{n-1} \tilde{f}_{k} \xi_{k} i e^{-\rho x_{n}}-\rho \tilde{f}_{n} e^{-\rho x_{n}}+C e^{h x_{n}}+ \\
& \quad+\frac{C}{h^{2}-\rho^{2}}\left(\lambda_{n n} \rho h-\rho \sum_{j=1}^{n-1} \lambda_{n j} \xi_{j} i+\lambda_{n n} h^{2}-\sum_{j=1}^{n-1} \lambda_{n j} \xi_{j} h i-\left(h^{2}-\rho^{2}\right)\right)=C e^{h x_{n}} .
\end{aligned}
$$

Преобразуем выражение, стоящее в скобках:

$$
\begin{gathered}
\frac{1}{h^{2}-\rho^{2}}\left(\lambda_{n n} \rho h-\rho i \sum_{j=1}^{n-1} \lambda_{n j} \xi_{j}+\lambda_{n n} h^{2}-h i \sum_{j=1}^{n-1} \lambda_{n j} \xi_{j}+\rho^{2}-h^{2}\right)= \\
=\frac{(h+\rho)\left(\lambda_{n n} h-\sum_{j=1}^{n-1} \lambda_{n j} \xi_{j} i-(h-\rho)\right)}{h^{2}-\rho^{2}}=\frac{h\left(\lambda_{n n}-1\right)-\sum_{j=1}^{n-1} \lambda_{n j} \xi_{j} i-\rho}{h-\rho}= \\
=\frac{h\left(\lambda_{n n}-1\right)-\sum_{j=1}^{n-1} \lambda_{n j} \xi_{j} i-\rho}{h-\rho} \times \frac{h\left(\lambda_{n n}-1\right)-\sum_{j=1}^{n-1} \lambda_{n j} \xi_{j} i+\rho}{h\left(\lambda_{n n}-1\right)-\sum_{j=1}^{n-1} \lambda_{n j} \xi_{j} i+\rho}= \\
=\frac{\left(\sum_{j=1}^{n-1} \lambda_{n j} \xi_{j} i\right)^{2}-\rho^{2}}{h^{2}\left(\lambda_{n n}-1\right)^{2}-2 \sum_{j=1}^{n-1} \lambda_{n j} \xi_{j} i h\left(\lambda_{n n}-1\right)}+\frac{(h-\rho)\left(h\left(\lambda_{n n}-1\right)-\sum_{j=1}^{n-1} \lambda_{n j} \xi_{j} i+\rho\right)}{(h-\rho)\left(h\left(\lambda_{n n}-1\right)-\sum_{j=1}^{n-1} \lambda_{n j} \xi_{j} i+\rho\right)}
\end{gathered}
$$




$$
=\frac{h^{2}\left(\lambda_{n n}-1\right)^{2}-\alpha i h\left(\lambda_{n n}-1\right)^{2}}{(h-\rho)\left(h\left(\lambda_{n n}-1\right)-\sum_{j=1}^{n-1} \lambda_{n j} \xi_{j} i+\rho\right)}+\frac{\left(\sum_{j=1}^{n-1} \lambda_{n j} \xi_{j} i\right)^{2}-\rho^{2}}{(h-\rho)\left(h\left(\lambda_{n n}-1\right)-\sum_{j=1}^{n-1} \lambda_{n j} \xi_{j} i+\rho\right)} .
$$

Подставляя это выражение в (14) и учитывая, что $-i h \alpha+h^{2}=\beta^{2}$, находим

$$
\frac{C}{(h-\rho) \gamma}\left(\left(\lambda_{n n}-1\right)^{2} \beta^{2}-\left(\sum_{j=1}^{n-1} \lambda_{n j} \xi_{j}\right)^{2}-\rho^{2}\right)=\sum_{j=1}^{n-1} \tilde{f}_{j} \xi_{j} i+\rho \tilde{f}_{n}
$$

или

$$
\frac{C}{(h-\rho) \gamma}\left(\frac{\left(\lambda_{n n}-1\right)^{2}-\left(\sum_{k, j=1}^{n-1} \lambda_{k j} \xi_{k} \xi_{j}-\sum_{k=1}^{n-1} \xi_{k}^{2}\right)}{\lambda_{n n}-1}-\left(\sum_{j=1}^{n-1} \lambda_{n j} \xi_{j}\right)^{2}-\sum_{k=1}^{n-1} \xi_{k}^{2}\right)=\sum_{j=1}^{n-1} \tilde{f}_{j} \xi_{j} i+\rho \tilde{f}_{n},
$$

где

$$
\gamma=h\left(\lambda_{n n}-1\right)-\sum_{j=1}^{n-1} \lambda_{n j} \xi_{j} i+\rho .
$$

Пусть $\tilde{w}=C\left(\xi_{1}, \ldots, \xi_{n-1}\right)$, т.е. $C\left(\xi_{1}, \ldots, \xi_{n-1}\right)$ - трансформанта Фурье некоторой функции $w\left(x_{1}, \ldots, x_{n-1}\right)$. Применив к $(15)$ обратное преобразование Фурье, получим для функции $w$ линейное уравнение второго порядка

$$
\lambda_{n n} \Delta w-\sum_{k, j=1}^{n-1}\left(\lambda_{k j}\left(\lambda_{n n}-1\right)-\lambda_{n k} \lambda_{n j}\right) \frac{\partial^{2} w}{\partial x_{k} \partial x_{j}}=F\left(x_{1}, \ldots, x_{n-1}\right),
$$

где $F\left(x_{1}, \ldots, x_{n-1}\right)$ - известная функция. Приведем это уравнение к каноническому виду. Возможны четыре случая.

I. Если уравнение (16) эллиптично во всем пространстве переменных $x_{1}, \ldots, x_{n-1}$, то, приведя его к каноническому виду, получим уравнение Пуассона

$$
\sum_{j=1}^{n-1} \frac{\partial^{2} w}{\partial \eta_{j}^{2}}=F\left(\eta_{1}, \ldots, \eta_{n-1}\right)
$$

Оно имеет единственное стремящееся к нулю на бесконечности решение (см. [3]). Следовательно, задача (3)-(4) разрешима, и решение ее единственно.

II. Если уравнение (16) гиперболично, имеем

$$
\sum_{j=1}^{n-2} \frac{\partial^{2} w}{\partial \eta_{j}^{2}}-\frac{\partial^{2} w}{\partial \eta_{n-1}^{2}}=F\left(\eta_{1}, \ldots, \eta_{n-1}\right)
$$

Однородной задаче Дирихле для системы (3) соответствует однородное уравнение

$$
\sum_{j=1}^{n-2} \frac{\partial^{2} w}{\partial \eta_{j}^{2}}-\frac{\partial^{2} w}{\partial \eta_{n-1}^{2}}=0
$$

Его решение ищем в виде

$$
w=v\left(\eta_{1}, \ldots, \eta_{n-2}\right) X\left(\eta_{n-1}\right)
$$

Подставляя $w$ из (19) в (18), получим два уравнения

$$
X^{\prime \prime}+\lambda^{2} X=0
$$




$$
\Delta v+\lambda^{2} v=0
$$

где $\lambda$-некоторая постоянная. Уравнение (20) имеет общее решение

$$
X=a_{k} \cos \lambda \eta_{n-1}+b_{k} \sin \lambda \eta_{n-1} .
$$

Решение уравнения (21) будем искать в виде

$$
v=P_{l}\left(\eta_{1}, \ldots, \eta_{n-2}\right) \cdot g(t),
$$

где $P_{l}\left(\eta_{1}, \ldots, \eta_{n-2}\right)$ - однородный гармонический полином степени $l, t=\sum_{j=1}^{n-2} \eta_{j}^{2}, g(t)$ - неизвестная функция, которую определим так, чтобы функция, определенная по формуле (22), была решением уравнения (21). Подставляя $v$ из (22) в (21), получим

$$
\Delta P_{l} \cdot g+\Delta g \cdot P_{l}+2 \sum_{i=1}^{n-2} \frac{\partial g}{\partial \eta_{i}} \cdot \frac{\partial P_{l}}{\partial \eta_{i}}+\lambda^{2} g P_{l}=0
$$

Так как

$$
\frac{\partial g}{\partial \eta_{i}}=g^{\prime} \cdot 2 \eta_{i}, \quad \frac{\partial^{2} g}{\partial \eta_{i}^{2}}=4 g^{\prime \prime} \cdot \eta_{i}^{2}+2 g^{\prime}, \quad \Delta P_{l}=0
$$

имеем

$$
4 g^{\prime \prime} P_{l} \cdot \sum_{i=1}^{n-2} \eta_{i}^{2}+2(n-2) g^{\prime} P_{l}+4 g^{\prime} \cdot \sum_{i=1}^{n-2} \eta_{i} \frac{\partial P_{l}}{\partial \eta_{i}}+\lambda^{2} g P_{l}=0
$$

По формуле Эйлера

$$
\sum_{i=1}^{n-2} \eta_{i} \frac{\partial P_{l}}{\partial \eta_{i}}=l \cdot P_{l}
$$

Тогда из последнего уравнения получим

$$
P_{l}\left(4 g^{\prime \prime} t+4 g^{\prime} l+2(n-2) g^{\prime}+\lambda^{2} g\right)=0
$$

или

$$
g^{\prime \prime} t+\left(l+\frac{n-2}{2}\right) g^{\prime}+\frac{\lambda^{2}}{4} g=0
$$

Решение этого уравнения будем искать в виде ряда $g=\sum_{k=0}^{\infty} c_{k} t^{k}$. Подставляя $g$ в уравнение (23), получаем рекуррентные соотношения

$$
c_{1}=\frac{-\lambda^{2} / 4}{1 \cdot\left(l+\frac{n-2}{2}\right)} \cdot c_{0}, \quad c_{2}=\frac{-\lambda^{2} / 4}{2 \cdot\left(1+l+\frac{n-2}{2}\right)} \cdot c_{1}, \quad \ldots, \quad c_{k+1}=\frac{-\lambda^{2} / 4}{(k+1)\left(k+l+\frac{n-2}{2}\right)} \cdot c_{k},
$$

или

$$
c_{k}=\frac{(-1)^{k}\left(\lambda^{2} / 4\right)^{k} c_{0}}{k !\left(l+\frac{n-2}{2}\right)\left(1+l+\frac{n-2}{2}\right) \cdots\left(k-1+l+\frac{n-2}{2}\right)}, \quad k=1,2, \ldots
$$

Пусть $c_{0}=1 / \Gamma\left(l+\frac{n-2}{2}\right) ;$ тогда

$$
c_{k}=\frac{(-1)^{k}\left(\lambda^{2} / 4\right)^{k}}{k ! \Gamma\left(k+l+\frac{n-2}{2}\right)}, \quad k=1,2, \ldots
$$

Итак,

$$
g=\sum_{k=0}^{\infty} \frac{(-1)^{k}\left(\lambda^{2} / 4\right)^{k} t^{k}}{k ! \Gamma\left(k+l+\frac{n-2}{2}\right)} .
$$


Учитывая, что

$$
J_{\nu}(x)=\sum_{k=0}^{\infty} \frac{(-1)^{k}}{\Gamma(k+\nu+1) \Gamma(k+1)} \cdot\left(\frac{x}{2}\right)^{2 k+\nu},
$$

где $J_{\nu}(x)$ - функция Бесселя первого рода, получим

$$
g(t)=\left(\frac{\lambda \sqrt{t}}{2}\right)^{-l-(n-4) / 2} J_{l+(n-4) / 2}\left(\frac{\lambda \sqrt{t}}{2}\right) .
$$

Из свойств функции Бесселя следует, что $f$ стремится к нулю на бесконечности по переменным $\eta_{1}, \ldots, \eta_{n-2}$, а функция $X$ ограничена по переменной $\eta_{n-1}$. Таким образом, уравнение (18) имеет бесконечно много решений стремящихся к нулю на бесконечности по переменным $\eta_{1}, \ldots, \eta_{n-2}$ и ограниченных по переменной $\eta_{n-1}$. Следовательно, однородная задача Дирихле для системы (3) имеет бесконечно много ограниченных, но не стремящихся к нулю на бесконечности решений.

III. Если уравнение (16) ультрагиперболично, то, приведя его к каноническому виду, получим

$$
\sum_{j=1}^{m-1} \frac{\partial^{2} w}{\partial \eta_{j}^{2}}-\sum_{j=m}^{n-1} \frac{\partial^{2} w}{\partial \eta_{j}^{2}}=F\left(\eta_{1}, \ldots, \eta_{n-1}\right), \quad 2<m<n-2 .
$$

Рассмотрим однородное уравнение, соответствующее (24):

$$
\sum_{j=1}^{m-1} \frac{\partial^{2} w}{\partial \eta_{j}^{2}}-\sum_{j=m}^{n-1} \frac{\partial^{2} w}{\partial \eta_{j}^{2}}=0 .
$$

Разделяя переменные по формуле

$$
w=X\left(\eta_{1}, \ldots, \eta_{m-1}\right) \cdot Y\left(\eta_{m}, \ldots, \eta_{n-1}\right)
$$

получим два уравнения

$$
\Delta X+\lambda^{2} X=0, \quad \Delta Y+\lambda^{2} Y=0 .
$$

Аналогично предыдущему находим

$$
\begin{aligned}
& X=P_{l_{1}}\left(\eta_{1}, \ldots, \eta_{m-1}\right)\left(\frac{\lambda \sqrt{t_{1}}}{2}\right)^{-l_{1}-(m-3) / 2} J_{l_{1}+(m-3) / 2}\left(\frac{\lambda \sqrt{t_{1}}}{2}\right), \\
& Y=P_{l_{2}}\left(\eta_{m}, \ldots, \eta_{n-1}\right)\left(\frac{\lambda \sqrt{t_{2}}}{2}\right)^{-l_{2}-(n-m-2) / 2} J_{l_{2}+(n-m-2) / 2}\left(\frac{\lambda \sqrt{t_{2}}}{2}\right),
\end{aligned}
$$

где $P_{l_{1}}$ и $P_{l_{2}}$ - однородные гармонические полиномы степеней $l_{1}$ и $l_{2}$ соответственно,

$$
t_{1}=\sum_{j=1}^{m-1} \eta_{j}^{2}, \quad t_{2}=\sum_{j=m}^{n-1} \eta_{j}^{2} .
$$

Однородное уравнение, соответствующее уравнению (24), а, следовательно, и однородная задача Дирихле для системы (3) имеет бесконечно много стремящихся к нулю на бесконечности решений. Заметим, что этот случай возможен только при $n>4$.

IV. Если уравнение (16) является уравнением параболического типа, то, приведя его к каноническому виду, имеем

$$
\sum_{j=1}^{m} \frac{\partial^{2} w}{\partial \eta_{j}^{2}}=F\left(\eta_{1}, \ldots, \eta_{n-1}\right), \quad 1<m<n-1 .
$$

В этом случае однородная задача имеет бесконечно много ограниченных, но не стремящихся к нулю на бесконечности решений.

Таким образом, справедлива следующая теорема. 
Теорема 1. Характер разрешимости задачи Дирихле для системы (3) в полупространстве $G=\left\{x_{n}>0\right\}$ определяется видом уравнения (16):

$$
\lambda_{n n} \Delta w-\sum_{k, j=1}^{n-1}\left(\lambda_{k j}\left(\lambda_{n n}-1\right)-\lambda_{n k} \lambda_{n j}\right) \frac{\partial^{2} w}{\partial x_{k} \partial x_{j}}=F\left(x_{1}, \ldots, x_{n-1}\right),
$$

которое рассматривается во всем пространстве переменных $x_{1}, \ldots, x_{n-1}$. Однородной задаче Дирихле соответствует однородное уравнение. Если это уравнение эллиптично, то рассматриваемая задача Дирихле имеет единственное решение; если уравнение (16) параболично или гиперболично, то однородная задача Дирихле для системы (3) имеет бесконечное множество ограниченных, но не стремящихся к нулю на бесконечности решений; если же уравнение (16) ультрагиперболично, то однородная задача имеет бесконечное множество стремящихся к нулю на бесконечности решений.

\section{СПИСОК ЛИТЕРАТУРЫ}

1. Бицадзе А. В. Некоторые классы уравнений в частных производных. - М.: Наука, 1981.

2. Вишик М. И. О сильно эллиптических системах дифференциальных уравнений// Мат. сб. - 1951. 29, № 3. - С. 615-676.

3. Курант Р. Уравнения с частными производными. - М.: Мир, 1964.

4. Янушаускас A. И. Граничные задачи для уравнений в частных производных и интегродифференциальные уравнения. - Иркутск: Изд-во Иркут. ун-та, 1997.

5. Янушаускас А. И. Задача о наклонной производной теории потенциала. - Новосибирск: Наука, 1985.

Головко Елена Анатольевна

Иркутский государственный университет

E-mail: elena-golovko@mail.ru 